\title{
When is a Field Theory a Generalized Free Field?
}

\author{
Klaus Baumann \\ Bell Laboratories, Murray Hill, New Jersey, USA, \\ and Max-Planck-Institut für Physik und Astrophysik, München, Federal Republic of Germany
}

Received April 8, 1975

\begin{abstract}
We show within a scalar relativistic quantum field theory that if either some even truncated $n$-point-function vanishes or some multiple commutator of the field operators is a C-number then the field is necessarily a generalized free field.
\end{abstract}

\section{I.}

The generalized free fields are well known examples for relativistic quantum field theories. Introduced in 1961 by Greenberg [1] they have been extensively studied since then. Of special interest was the question under what conditions a field theory is necessarily a generalized free field. As shown by Dell'Antonio [2], Robinson [3] and Greenberg [4] such a sufficient condition is that the support of the field in momentum space excludes certain domains. Robinson [5] gave another criterion namely the vanishing of the truncated $n$-point-functions beyond some $N$. In this note we shall strengthen this result and prove - with entirely different methods than Robinson - that if an arbitrary even truncated Wightman function vanishes the field must be a generalized free field.

\section{II.}

We consider a relativistic scalar field $A(x)$ which we assume to fulfill Wightman's axioms $[6,7]$. We denote the vacuum state by $\Omega$ and the $n$-point-functions by

$$
\mathscr{W}_{n}\left(x_{1}, \ldots, x_{n}\right)=\left(\Omega, A\left(x_{1}\right), \ldots, A\left(x_{n}\right) \Omega\right) .
$$

Without restriction we can assume $(\Omega, A(x) \Omega)=0$. The truncated $n$-point-functions [7] are recursively defined by

$$
\begin{aligned}
& \mathscr{W}_{1}(x)=\mathscr{W}_{1}^{T}(x) \quad\left[\text { and therefore } \mathscr{W}_{1}^{T}(x)=0\right] \\
& \mathscr{W}_{n}\left(x_{1}, \ldots, x_{n}\right)= \sum_{\text {partitions }} \mathscr{W}_{r_{1}}^{T}\left(x_{l_{1}(1)} \ldots x_{l_{1}\left(r_{1}\right)}\right) \\
& \times \mathscr{W}_{r_{2}}^{T}\left(x_{l_{2}(1)} \ldots x_{l_{2}\left(r_{2}\right)}\right) \ldots \mathscr{W}_{r_{s}}^{T}\left(x_{l_{s}(1)} \ldots x_{l_{s}\left(r_{s}\right)}\right) .
\end{aligned}
$$

Now we are able to formulate

Theorem 1. If $\mathscr{W}_{2 n}^{T}\left(x_{1} \ldots x_{2 n}\right) \equiv 0$ for some $n \geqq 2$ then

$\left[\ldots\left[A\left(x_{1}\right), A\left(x_{2}\right)\right] \ldots A\left(x_{n}\right)\right]=\left(\Omega,\left[\ldots\left[A\left(x_{1}\right), A\left(x_{2}\right)\right] \ldots A\left(x_{n}\right)\right] \Omega\right)^{T} \cdot \mathbb{1}$. 
Proof. One can easily prove by induction that

$$
\begin{aligned}
(\Omega, & {\left[\ldots\left[A\left(x_{1}\right), A\left(x_{2}\right)\right] \ldots A\left(x_{n}\right)\right] } \\
& \times\left[A\left(x_{n+1}\right)\left[A\left(x_{n+2}\right) \ldots\left[A\left(x_{2 n-1}\right), A\left(x_{2 n}\right)\right] \ldots\right] \Omega\right) \\
= & \left(\Omega,\left[\ldots\left[A\left(x_{1}\right), A\left(x_{2}\right)\right] \ldots A\left(x_{n}\right)\right]\left[A\left(x_{n+1}\right) \ldots\left[A\left(x_{2 n-1}\right), A\left(x_{2 n}\right)\right] \ldots\right] \Omega\right)^{T} \\
& +\left(\Omega,\left[\ldots\left[A\left(x_{1}\right), A\left(x_{2}\right)\right] \ldots A\left(x_{n}\right)\right] \Omega\right)^{T} \\
& \left.\times\left(\Omega,\left[A\left(x_{n+1}\right) \ldots\left[A\left(x_{2 n-1}\right), A_{2 n}\right)\right] \ldots\right] \Omega\right)^{T} .
\end{aligned}
$$

But $\mathscr{W}_{2 n}^{T}\left(x_{1}, \ldots, x_{2 n}\right) \equiv 0$ and therefore we get

$$
\begin{aligned}
& \left\|\left[\ldots\left[A\left(x_{1}\right), A\left(x_{2}\right)\right] \ldots A\left(x_{n}\right)\right] \Omega\right\|^{2} \\
& =\left|\left(\Omega,\left[\ldots\left[A\left(x_{1}\right), A\left(x_{2}\right)\right] \ldots A\left(x_{n}\right)\right] \Omega\right)^{T}\right|^{2} .
\end{aligned}
$$

This implies

$$
\begin{aligned}
& {\left[\ldots\left[A\left(x_{1}\right), A\left(x_{2}\right)\right] \ldots A\left(x_{n}\right)\right] \Omega} \\
& =\left(\Omega,\left[\ldots\left[A\left(x_{1}\right), A\left(x_{2}\right)\right] \ldots A\left(x_{n}\right)\right] \Omega\right)^{T} \cdot \Omega
\end{aligned}
$$

and by locality (see Jost [7], p. 99) we get Theorem 1.

\section{III.}

Our main result is contained in

Theorem 2. If $\left[\ldots\left[A\left(x_{1}\right), A\left(x_{2}\right)\right] \ldots A\left(x_{n}\right)\right]=c \cdot \mathbb{1}$ then $A(x)$ is a generalized free field.

Proof. a) By a theorem of Robinson [3] and Greenberg [4] it is sufficient to show that $\widetilde{A}(p) \equiv 0$ for $p^{2}<0$ (i.e. for all spacelike momentum $p$ ). For this purpose let us introduce the following space of test functions: Define

$$
\begin{aligned}
S: & =\left\{x \in \mathbb{R}^{4} \mid x^{2}<0\right\} \quad \text { "spacelike region" } \\
\tilde{\mathscr{D}}(S) & :=\left\{f \in \mathscr{S}\left(\mathbb{R}^{4}\right) \mid \tilde{f}(p)=\int e^{i p x} f(x) d^{4} x \in \mathscr{D}(S)\right\}
\end{aligned}
$$

"space of test functions whose Fourier transforms have support only in the spacelike region".

We note that with $f$ also $\bar{f}$ is in $\tilde{\mathscr{D}}(S)$. The spectrum condition $[6,7]$ implies

$A(f) \Omega=0 \quad$ if $\quad f \in \tilde{\mathscr{D}}(S)$.

b) For a vector $\Phi$ in the dense domain of the field operators $A(f)$ we have the inequality

$$
\|A(f) \Phi\| \leqq\|\Phi\|^{1-\frac{1}{2^{m}}}\|\underbrace{A(\bar{f}) A(f) \ldots A(\bar{f}) A(f) \Phi}_{2^{m} \text { operators }}\|^{\frac{1}{2^{m}}}
$$

By this $A\left(f_{1}\right) \ldots A\left(f_{n}\right) \Phi=0$ for all $f_{1}, \ldots, f_{n} \in \tilde{\mathscr{D}}(S)$ implies $A(f) \Phi=0$ for all $f \in \tilde{\mathscr{D}}(S)$.

c) Proposition. $A(f) A\left(g_{1}\right) \ldots A\left(g_{l}\right) \Omega=0$ for all $l$ and all test functions $f \in \tilde{\mathscr{D}}(S)$ and $g_{1}, \ldots, g_{l} \in \mathscr{S}\left(\mathbb{R}^{4}\right)$. 
Proof. By induction:

$A(f) \Omega=0$ for $f \in \tilde{\mathscr{D}}(S) \quad$ by the spectrum condition.

Now assume $A(f) A\left(g_{1}\right) \ldots A\left(g_{l}\right) \Omega=0$ for all $f \in \tilde{\mathscr{D}}(S)$ and all $g_{1}, \ldots, g_{l} \in \mathscr{S}\left(\mathbb{R}^{4}\right)$ then for $f_{1}, \ldots, f_{n} \in \tilde{\mathscr{D}}(S)$

$$
\begin{aligned}
& A\left(f_{1}\right) \ldots A\left(f_{n}\right) A\left(g_{1}\right) \ldots A\left(g_{l+1}\right) \Omega \\
& =A\left(f_{1}\right) \ldots A\left(f_{n-1}\right)\left[A\left(f_{n}\right), A\left(g_{1}\right)\right] A\left(g_{1}\right) \ldots A\left(g_{l+1}\right) \Omega \\
& =A\left(f_{1}\right) \ldots\left[A\left(f_{n-1}\right)\left[A\left(f_{n}\right), A\left(g_{1}\right)\right]\right] A\left(g_{2}\right) \ldots A\left(g_{l+1}\right) \Omega \\
& \vdots \\
& \left.\quad[\underbrace{A\left(f_{1}\right)\left[A ( f _ { 2 } ) \ldots \left[A\left(f_{n}\right), A\left(g_{1}\right)\right.\right.}_{=0}] \ldots\right] A\left(g_{2}\right) \ldots A\left(g_{l+1}\right) \Omega=0
\end{aligned}
$$

because by assumption every multiple commutator with $n+1$ field operators vanishes.

d) The vectors $A\left(g_{1}\right) \ldots A\left(g_{l}\right) \Omega$ for all $l$ and $g_{1}, \ldots, g_{l} \in \mathscr{S}\left(\mathbb{R}^{4}\right)$ form a dense set in the domain of $A(f)$ and therefore $A(f) \equiv 0$ for $f \in \tilde{\mathscr{D}}(S)$. This proves our Theorem 2.

Remark. It one looks into the proofs of the above mentioned theorem of Robinson [3] and Greenberg [4] one immediately realizes that it is already sufficient to know that

$$
A(f) A(g) \Omega=0 \text { for all } f \in \tilde{\mathscr{D}}(S) \text { and } g \in \mathscr{S}\left(\mathbb{R}^{4}\right) .
$$

But by the inequality given in part (b) we can easily formulate this as a condition imposed on an arbitrary even truncated Wightman function, namely

$$
\mathscr{W}_{2 n}^{T}\left(g, f_{1}, \ldots, f_{2 n-2}, h\right)=0 \text { for all } g, h \in \mathscr{S}\left(\mathbb{R}^{4}\right) \text { and } f_{1}, \ldots, f_{2 n-2} \in \tilde{\mathscr{D}}(S) .
$$

Conclusion. By combining these two theorems we have shown that in an interacting relativistic quantum field theory every even truncated Wightman function must not vanish identically.

Acknowledgement. I want to thank Dr. H. Narnhofer for many helpful discussions.

\section{References}

1. Greenberg, O.W.: Annals of Physics 16, 158 (1961)

2. Dell'Antonio, G.F.: J. Math. Phys. 2, 759 (1961)

3. Robinson, D.W.: Helv. Phys. Acta 35, 403 (1962)

4. Greenberg, O. W.: J. Math. Phys. 3, 859 (1962)

5. Robinson, D. W.: Commun. math. Phys. 1, 89 (1965)

6. Streater, R.F., Wightman, A.S.: PCT, Spin and Statistics and All That. New York: Benjamin 1964

7. Jost, R.: The General Theory of Quantized Fields, AMS, Providence, 1965 
